\title{
Características del uso de Sildenafil en una muestra de pacientes a nivel de farmacias comunitarias de Concepción, Chile
}

\author{
Sigrid Mennickent $\mathrm{C}^{\mathrm{la}}$, Libna Zurita $\mathrm{Z}^{\mathrm{b}}$, Mario Vega $\mathrm{H}^{2 \mathrm{c}}$, \\ G uiljardy Villegas $\mathbf{M}^{\mathrm{b}}$. \\ Features of subjects purchasing \\ Sildenafil at community pharmacies \\ in Concepción, Chile
}

Background: Erectile dysfunction is a public health problem. Millions of men worldwide experience this condition, affecting their quality of life. Sildenafil, a phosphodiesterase type 5 inhibitor, is the treatment of choice for erectile dysfunction. Aim: To study the characteristics of Sildenafil use among costumers of community pharmacies in Concepcion Chile. Material and methods: A structured interview about Sildenafil use was applied to subjects purchasing the drug in community pharmacies and that consented to participate. Results: One hundred thirty eight males were studied. Forty six percent of patients were fifty years old or older. Most (47.1\%) used Sildenafil for one year or more and $60.5 \%$ had no adverse events. Among those experiencing side effects, headache was experienced by $38.9 \%$, flushing by $16.6 \%$ and gastrointestinal effects by $16.7 \%$. Ninety percent of patients had a good evaluation of the drug in terms of benefits and tolerance. Thirty two percent of the subjects, had diseases associated to erectile dysfunction. Among these, $45 \%$ had diabetes mellitus, $29.5 \%$ had hypertension, and $18.2 \%$ had prostatic diseases. In $54 \%$ of cases, the drug was prescribed by a physician. Twenty two percent of patients experienced changes in the effect of the drug when it was simultaneously used with other drugs or meals. Thirty four percent of patients used other drugs. Among these, $43.3 \%$ were using antihypertensives, and $26.7 \%$ were using antidiabetic drugs. Conclusions: Considering the elevated age and the number of associated disease of patients using Sildenafil, the use of the drug under medical prescription must be emphasized to avoid untoward effects (Rev Méd Chile 2005; 133: 667-73).

(Key Words: Impotence; Prescriptions, drugs; Sildenafil)

Recibido el 11 de junio, 2004. Aceptado en versión corregida el 5 de abril, 2005.

${ }^{1}$ Departamento de Farmacia, Facultad de Farmacia, Universidad de Concepción. ${ }^{2}$ Departamento de Bromatología, Nutrición y Dietética, Facultad de Farmacia, Universidad de Concepción, Concepción, Chile.

aQuímico Farmacéutico, Magíster en Ciencias Farmacéuticas.

${ }^{\mathrm{b}} \mathrm{Químico} \mathrm{Farmacéutico.}$

'Químico Farmacéutico, Magíster en Nutrición Humana.

Correspondencia a: Sigrid Mennickent C. Departamento de Farmacia, Universidad de Concepción. Casilla 237, Concepción, Chile. Fono: 56-41-204523. Fax: 56-41-207086.

E-mail: smennick@udec.cl 
$E^{\mathrm{n}}$ 1993, The National Institute of Health Con sensus Conference de los Estados Unidos de Norteamérica (USA), recomendó que la palabra "impotencia" fuese reemplazada por "disfunción eréctil", término menos peyorativo y con menor connotación psicológica. Actualmente, esta recomendación ha sido adoptada mundialmente.

Una gran proporción de hombres experimenta disfunción eréctil en algún período de sus vidas, la que puede ser transitoria o permanente, completa o parcial. Frecuentes episodios de disfunción eréctil pueden causar problemas emocionales y de relación de pareja y, a menudo, llevan a una disminución de la autoestima ${ }^{1}$.

La disfunción eréctil (DE) se define como la inhabilidad consistente para alcanzar y mantener una erección del pene satisfactoria para el coito, en al menos $50 \%$ de los intentos ${ }^{1-5}$. Sólo en USA afecta a 30 millones de hombres, aproximadamente, con más de 900.000 nuevos casos cada año y se estima que, a nivel mundial, alrededor de 150 millones sufren de alguna forma de esta patología. $\mathrm{Su}$ incidencia aumenta con la edad, en hombres con diabetes mellitus y en pacientes con enfermedades vasculares ${ }^{2,6-8}$.

El origen de la disfunción eréctil es multifactorial. En general, cualquier patología o factor que afecte la irrigación sanguínea, inervación neural o el balance endocrino de los órganos sexuales resultará en una disfunción eréctil. Otras causas son psicológicas (estados depresivos, de preocupación, de culpa, estrés y ansiedad) y también la utilización de algunos medicamentos, como betabloqueadores, hidroclorotiazida, antisicóticos, antidepresivos, digoxina, antagonistas androgénicos, algunos antagonistas H2, antineoplásicos, entre otros. El abuso del alcohol y la nicotina también son causales de disfunción eréctil ${ }^{3}$.

La estrategia terapéutica se determina por la etiología y la severidad de la enfermedad ${ }^{3-5}$. Entre las alternativas se encuentran el tratamiento psicológico, terapia intracavernosa (Alprostadil), dispositivos mecánicos, intervenciones quirúrgicas y terapia oral. El tratamiento vía oral es el más utilizado y entre los medicamentos existentes para ser administrados por esta vía se encuentran apomorfina, yohimbina, andrógenos e inhibidores de la fosfodiesterasa (PDE) tipo 5 (Sildenafil, Vardenafil, Tadalafil, Tardenafil). El más utilizado en la actualidad es Sildenafil.
Los inhibidores de la PDE-5 actúan aumentando el efecto del óxido nítrico, al inhibir la actividad de la isoforma de la enzima que predomina en el pene. Esto produce una disminución de la degradación del GMP cíclico, responsable de la relajación de la musculatura lisa del cuerpo cavernoso, lo que se manifiesta en una erección. Estos fármacos no poseen efecto relajante directo sobre el cuerpo cavernoso humano aislado ni efecto en ausencia de estímulo sexual.

Sildenafil es rápidamente absorbido luego de su administración, presentando una biodisponibilidad de alrededor de $40 \%$. La concentración plasmática máxima se alcanza en alrededor de una hora cuando se administra en ayunas, y en alrededor de dos horas cuando se administra junto a comidas ricas en grasas. El fármaco es metabolizado en el hígado a un metabolito activo. Se excreta preferentemente por las heces (alrededor de $80 \%$ ) y también por la orina (alrededor de 13\%) ${ }^{7}$. Tanto el fármaco original como su metabolito activo presentan una vida media de 3-5 horas $3,7,9,10$. El clearance es menor en hombres mayores de 65 años y en pacientes con falla hepática o renal severa ${ }^{7}$.

El objetivo del presente estudio fue evaluar las características del uso de Sildenafil en una muestra de pacientes que despachan este medicamento en farmacia comunitaria.

\section{MATERIAL Y MÉTODO}

Se realizó un registro acerca de las características del uso de Sildenafil durante los meses de noviembre y diciembre de 2004 mediante un cuestionario estructurado, el que fue aplicado en ocho farmacias comunitarias de la ciudad de Concepción, Chile, a todos los pacientes que despacharon Sildenafil y que consintieron en participar en el estudio $(n=138)$, sin consideraciones especiales de número o tipo de pacientes. El único criterio de inclusión fue tener edad de 18 años o más.

El promedio de pacientes que despacharon Sildenafil en las farmacias consideradas, durante el año 2004, fue de 66 mensualmente. Las farmacias consideradas en el estudio, se eligieron de forma tal que representaran diferentes estratos socio-económicos y diferente afluencia de público. El número de farmacias participantes $(\mathrm{n}=8)$ se determinó de manera que representara a $10 \%$ del total de farmacias 
comunitarias alopáticas existentes en Concepción $(n=74)$, para garantizar que los resultados del estudio fuesen representativos del total de farmacias de la ciudad. El cuestionario aplicado a los pacientes incluyó edad de éstos, duración del tratamiento, tolerancia al tratamiento (en caso de presentar efectos adversos, se les preguntó cuáles fueron los predominantes), evaluación del tratamiento por parte de los pacientes (en cuanto a efectividad y tolerancia), y si el medicamento fue recetado por un médico (en caso de ser así, se consignó la especialidad del médico. De no haber sido prescrito por un médico, se determinó la fuente de información utilizada). También se consignó si los pacientes presentaban alguna enfermedad concomitante y cuáles eran las de mayor prevalencia. Se incluyeron, además, datos acerca de interacciones medicamentosas o alimentarias, y si los pacientes utilizaban algún otro medicamento en forma habitual.

La aplicación de los cuestionarios fue realizada por los químico farmacéuticos de las farmacias consideradas. Las respuestas fueron anónimas y confidenciales.

La evaluación de los datos se realizó mediante el software Excel.

\section{RESULTADOS}

La muestra estuvo constituida por 138 pacientes. El grupo etario que más despachó Sildenafil fue el de más de 50 años (46,38\%), seguido por los grupos comprendidos entre los 31 y 40 años $(20,30 \%)$ y entre los 41 y 50 años (19,56\%) (Figura 1).

El mayor porcentaje de pacientes que utilizaron Sildenafil lo hacía por más de un año $(47,10 \%)$, y el porcentaje más bajo $(11,59 \%)$ correspondió a pacientes que llevaban una terapia de menos de un mes (Figura 2). El 53,62\% de los pacientes encuestados utilizaron Sildenafil como tratamiento para la disfunción eréctil, por prescripción médica. El otro segmento lo hizo principalmente por recomendación de un amigo (21,74\%) o por consejo en la farmacia (17,39\%). El 7,25\% lo hizo por iniciativa propia (Figura 3). En cuanto a los pacientes que despacharon el medicamento por prescripción médica, $72 \%$ consultó a un médico general, $12 \%$ a un urólogo, 8\% a un cardiólogo, y 8\% no recordó o no supo definir la especialidad del médico que le recomendó la terapia (Figura 4). El 31,88\% de los

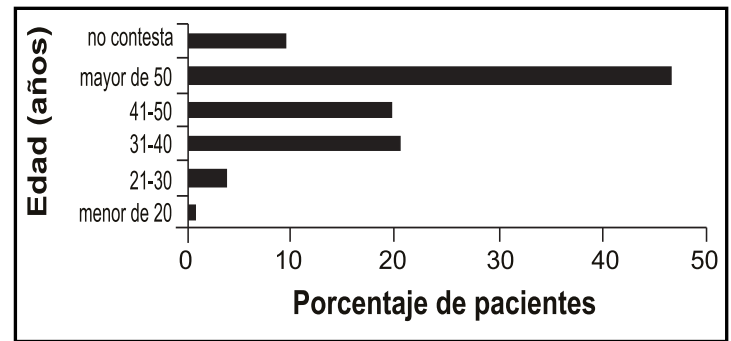

Figura 1. Distribución porcentual del consumo de Sildenafil según grupo etario.

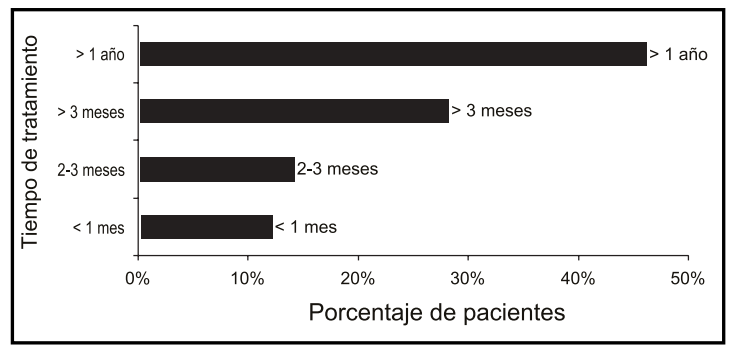

FIGURA 2. Distribución porcentual por tiempo de tratamiento con Sildenafil.

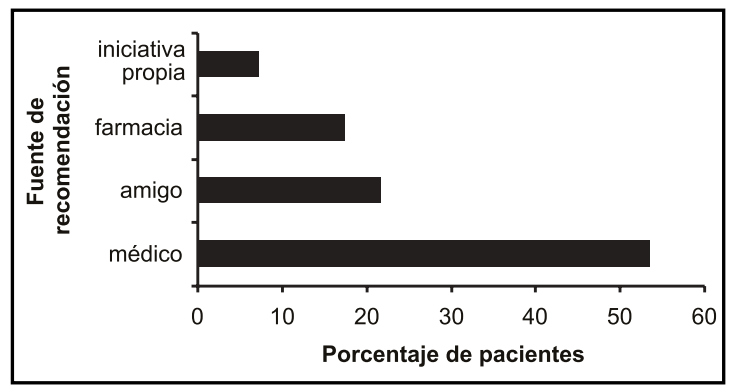

FIgURA 3. Información porcentual de fuente de recomendación de Sildenafil como terapia para la disfunción eréctil.

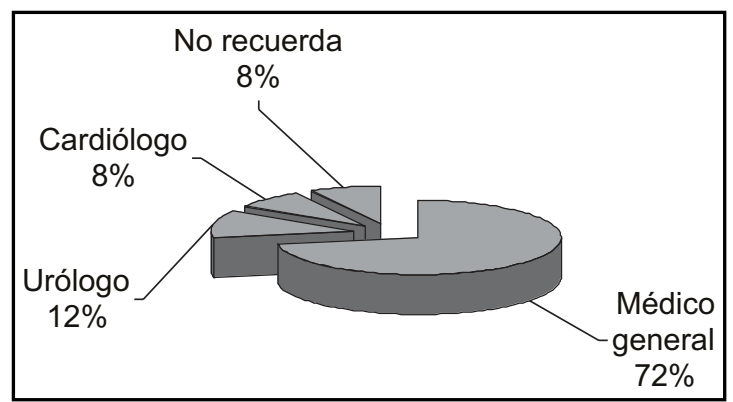

FIgURA 4. Distribución porcentual de especialidades médicas en la prescripción de Sildenafil. 


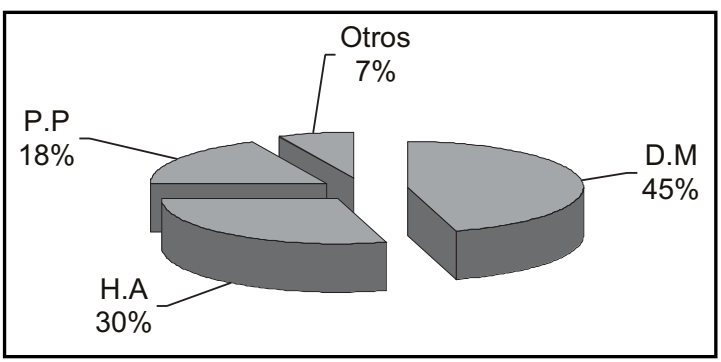

FIgURA 5. Distribución porcentual de patologías concomitantes a la disfunción eréctil. Universo: todos los pacientes con alguna otra patología además de DE.

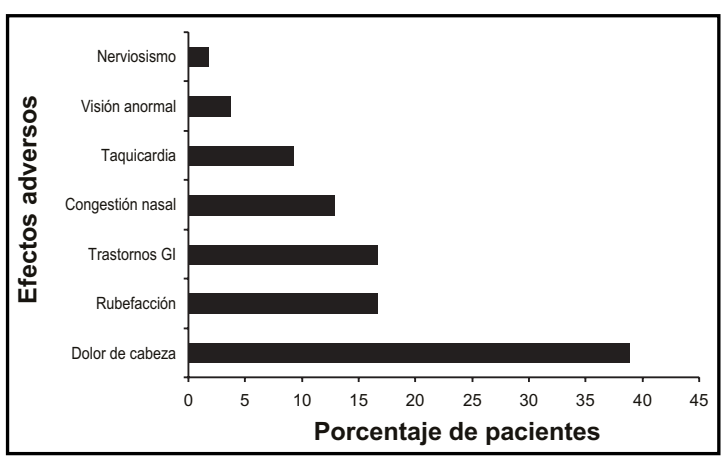

FIgURA 6. Distribución porcentual de efectos adversos producidos con la administración de Sildenafil. Universo: todos los pacientes que presentaron reacciones adversas.

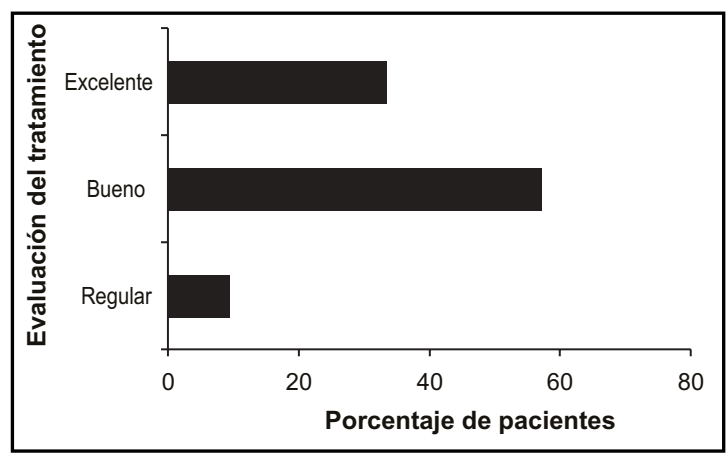

FiguRA 7. Evaluación del tratamiento con Sildenafil por parte de los pacientes. pacientes mencionó presentar alguna patología concomitante a la disfunción eréctil. La más frecuente fue la diabetes mellitus (DM), que constituyó $45,45 \%$ de las enfermedades declaradas. Otras patologías fueron hipertensión arterial (HA) (29,54\%) y alteraciones prostáticas (PP) (18,18\%) (Figura 5). El 64,49\% manifestó no presentar otra patología, y 5,07\% no contestó.

En cuanto a la tolerancia al tratamiento, $60,49 \%$ de los pacientes no presentó efectos adversos con la administración de Sildenafil. El 39,13\% manifestó haber sufrido algún efecto indeseable, y 1,45\% no contestó. Entre los pacientes que presentaron reacciones adversas, la más frecuente fue dolor de cabeza (38,89\%), seguido de rubefacción (16,67\%), efectos propios de la vasodilatación producida por Sildenafil. El 16,67\% $(n=9)$ de los pacientes que sufrieron efectos indeseables manifestó presentar trastornos gastrointestinales con el uso del fármaco, distribuyéndose en diarrea $(n=5)$, dolor estomacal $(n=2)$ y acidez/flatulencia $(\mathrm{n}=2)$ (Figura 6).

En cuanto a la evaluación de la terapia, la mayoría de los pacientes la calificó como buena (57,25\%) o excelente (33,33\%). El 9,42\% contestó que el tratamiento fue regular, y ningún paciente lo evaluó como malo (Figura 7).

En relación a posibles interacciones, $21,59 \%$ de los pacientes mencionó haber advertido alguna diferencia en el efecto de Sildenafil al administrarlo junto a otros medicamentos o a alimentos. De estos pacientes, 63,16\% manifestó una disminución del efecto del medicamento al ingerir alcohol, al igual que un aumento en la sensación de calor, y 31,58\% mencionó haber notado diferencias en el efecto del medicamento al ingerir comidas grasas. Un paciente manifestó una disminución del efecto de Sildenafil al administrarlo junto a una asociación de cafeína y efedrina (Figura 8).

De los pacientes encuestados, 34,09\% utilizaba otro(s) medicamento(s) en forma habitual, además de Sildenafil. De éstos, 43,33\% correspondió a antihipertensivos, especialmente a inhibidores de la enzima convertidora de angiotensina (ECA); 26,67\% a antidiabéticos, especialmente hipoglicemiantes orales (sólo un paciente utilizaba insulina), 13,33\% a antihistamínicos, y 13,33\% a analgésicos antiinflamatorios no-esteroidales (Figura 9). 


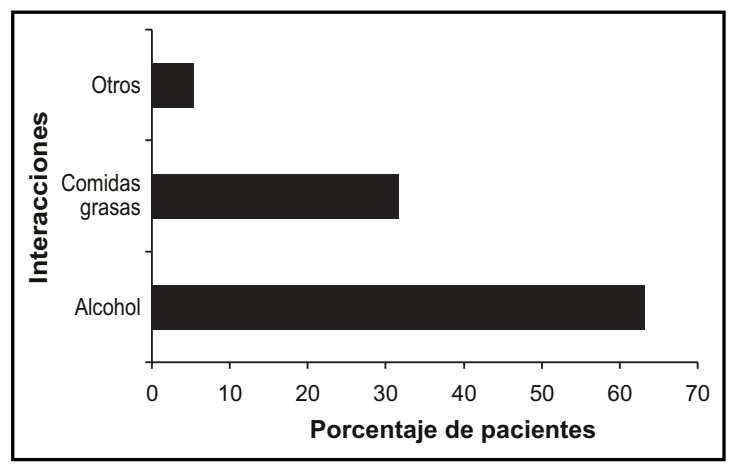

FIGURA 8. Interacciones con Sildenafil. Universo: todos los pacientes que manifestaron presentar alguna interacción.

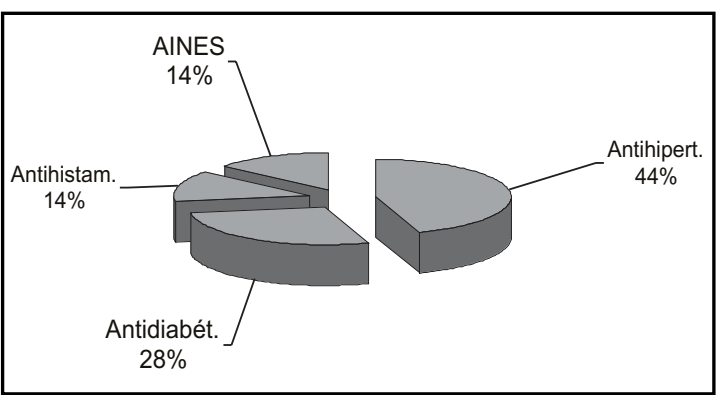

Figura 9. Medicamentos de uso habitual en la muestra de pacientes, además de Sildenafil. Universo: todos los pacientes que utilizaban otros medicamentos concomitantemente.

AINES= antiinflamatorios no-esteroidales

\section{DisCUSIÓN}

El promedio de despacho de este medicamento en las farmacias consideradas en el estudio, fue de $66 \mathrm{al} \mathrm{mes.} \mathrm{Entre} \mathrm{los} \mathrm{tres} \mathrm{inhibidores} \mathrm{de} \mathrm{fosfodies-}$ terasa-5 existentes en el mercado farmacéutico chileno, el Sildenafil tuvo una salida de alrededor de $91 \%-95 \%$ en las farmacias consideradas.

La muestra para este estudio estuvo constituida por 138 pacientes varones mayores de 18 años, que compraron el medicamento y que estuvieron de acuerdo en contestar el cuestionario destinado a conocer las características del uso de Sildenafil en pacientes a nivel de farmacia comunitaria. En todas las farmacias en las que se realizó el estudio hubo bastante reticencia por parte de los pacien- tes a entregar datos relacionados con el uso del medicamento.

Tan solo 5 de cada 10 hombres que usan Sildenafil, lo hacen por prescripción médica, de los cuales $72 \%$ es indicado por un médico general. La otra mitad de individuos que utilizan el fármaco lo hacen por recomendación de una persona no calificada para ello, lo que puede explicarse por la fuerte publicidad que existe de este medicamento. Esto es aún más preocupante debido a que la mayoría de los pacientes utiliza este fármaco por largo tiempo (47,10\% por más de un año), es de edad avanzada (46,38\% mayor de 50 años) y un porcentaje importante $(31,88 \%)$ presenta además otra patología, lo que suma otro factor de riesgo, cual es el uso habitual de otro(s) medicamento(s) aparte de Sildenafil (34,09\%). Todo esto lleva a mayor riesgo de reacciones adversas, interacciones, enmascaramiento de signos o síntomas de algunas patologías o empeoramiento de las ya existentes.

Un número importante de los pacientes que utilizaban el medicamento por más de un año habían obtenido recomendación de la terapia en la farmacia 0 a través de un amigo. Esto implica que no hay controles médicos de por medio que evalúen el estado fisiológico de los individuos.

El 60,49\% de los pacientes manifiestó no haber presentado episodios de reacciones adversas con el uso de Sildenafil; sin embargo 39,13\% sufrió algún efecto indeseable. De éstos, los predominantes fueron dolor de cabeza y rubefacción, que juntos suman $55,56 \%$ de las reacciones adversas declaradas, efectos propios de la vasodilatación producida por el medicamento. El 16,67\% de los pacientes que presentaron efectos indeseables manifestó trastornos gastrointestinales y 12,96\% describió haber tenido congestión nasal. Estos efectos se correlacionan con los encontrados en literatura ${ }^{6,7,11}$. El 3,70\% describió presentar visión anormal como efecto lateral, lo que se explica debido a que Sildenafil inhibe también a la PDE 6, aunque débilmente. Esta isoenzima interviene en la transmisión de señales de fotorreceptores, y por ello se le ha vinculado con alteraciones visuales, especialmente con cambios en la percepción de colores o en el brillo de la luz ${ }^{10}$.

En algunos casos un mismo paciente presentaba más de un efecto adverso. 
El 9,26\% de los pacientes que sufrieron reacciones adversas presentó taquicardia, lo cual no debiera subestimarse, ya que persisten dudas acerca del niesgo cardiovascular asociado a Sildenafil. Efectos menores relacionados con vasodilatación, como cefalea y rubor son relativamente frecuentes. Sin embargo, en pacientes con factores de riesgo cardiovascular preexistentes, debiera evaluarse con mayor atención el uso de este fármaco. En 1998 se informó a la Food and Drug Administration de los Estados Unidos de Norteamérica (FDA) de 69 decesos de pacientes que utilizaban Sildenafil: 2 de ellos a causa de accidente vascular cerebral y 46 por algún otro episodio cardiovascular. En pacientes que toman además nitratos, presentan riesgo de hipotensión potencialmente fatal lo que pudo haber condicionado alguno de estos casos $^{11}$. Por esto, cuando empezó a comercializarse en Chile, bajo el registrado Viagra ${ }^{\circledR}$, la condición de venta de este medicamento fue bajo receta retenida (Res. 4.274, $4.275 \mathrm{y}$ 4.276 del ISP, del 10-09-1998). Sin embargo, mediante la Resolución 4.471, se cambió su condición de venta a receta médica simple, debido a ausencia, a la fecha, de reportes de efectos adversos serios.

Se debe destacar también que, la mayor cantidad de individuos que utilizan Sildenafil para la terapia de disfunción eréctil son mayores de 50 años, lo que era de esperar $5,8,9,12,13$, pero llama la atención que el segundo y el tercer grupo etario que lo utiliza son los pacientes entre 31 y 40 años y aquellos entre 41 y 50 años, lo que rompe un mito que relaciona las patologías sexuales con la edad avanzada. Hubo un sólo paciente menor de 20 años, quien manifestó usarlo debido a la propaganda existente. Este paciente lo utilizaba por más de un año, y presentaba taquicardia, congestión nasal y diarrea como efectos laterales.

El 31,88\% de los pacientes manifestaron presentar alguna patología concomitante a la disfunción eréctil. De éstos, $45,40 \%$ correspondió a diabetes mellitus, lo que no resulta sorprendente, ya que la prevalencia de esta patología entre hombres con diabetes se encuentra entre $35 \%$ y $60 \% 3-5,8,12,13$. Otras enfermedades también importantes en la etiología de la disfunción eréctil, son la hipertensión arterial $(29,54 \%$ de los pacientes que tenían otra patología) y otras enfermedades cardiovasculares, alteraciones hepáticas, insuficiencia renal, alcoholismo crónico y cáncer de próstata 3,4,12,13. Sildenafil es una terapia que tiene una apreciación y evaluación de sus usuarios bastante positiva. El $57,25 \%$ de los pacientes considera a Sildenafil como una buena terapia, y 33,33\% la califica como excelente. En general, éstos últimos fueron pacientes que no presentaban efectos adversos, ni otras patologías, ni observaron interacciones con el uso del medicamento. Un porcentaje importante de pacientes presentó interacción con alcohol o con comidas grasas. Esto último se explica porque la absorción de Sildenafil se retrasa al administrarlo junto a este tipo de alimentos ${ }^{7}$. Debiera advertirse a los pacientes que deben consumir el fármaco $1 \mathrm{~h}$ antes o $2 \mathrm{~h}$ después de las comidas. En cuanto al alcohol, éste de por sí es un factor causante de disfunción eréctil.

También hay que tener cuidado con aquellos pacientes que utilizan concomitantemente medicamentos o alimentos que interfieran con el sistema citocromo CYP3A4, ruta de metabolización de Sildenafil. Así, compuestos que inhiban este sistema (como macrólidos, azoles, inhibidores de proteasas, jugo de pomelo) aumentarán los niveles plasmáticos de Sildenafil. Al contrario, si se trata de compuestos que estimulen el CYP3A4 (como rifampicina, fenobarbital, fenitoína, carbamazepina) disminuirán las concentraciones plasmáticas de Sildenafil y, por lo tanto, su eficacia?.

El 34,09\% de los pacientes encuestados utilizaban algún medicamento en forma simultánea a Sildenafil. De éstos, el mayor porcentaje correspondió a fármacos antihipertensivos, lo cual se correlaciona con la edad de la mayoría de los pacientes y con la etiología de la disfunción eréctil. El 26,67\% utilizaba medicamentos para la diabetes mellitus, lo cual también se relaciona con las causas de DE.

Las especialidades farmacéuticas de mayor salida fueron las de $50 \mathrm{mg}$ de dosificación, lo que se explica porque la dosis estándar aconsejada es de $50 \mathrm{mg}$, 1 hora antes del coito, aunque puede administrarse entre media hora y 4 horas antes de la actividad sexual. El rango posológico varía entre $25 \mathrm{mg}$ y $100 \mathrm{mg}$, de acuerdo a la eficacia y tolerancia, siempre una vez al día?.

Algunos pacientes $(n=6)$ despacharon especialidades de 1 forma farmacéutica, lo que puede deberse a que personas de mayor edad tienen relaciones sexuales esporádicas.

Se puede concluir que Sildenafil es un medicamento ampliamente utilizado en el tratamiento de 
la disfunción eréctil con muy buenos resultados y buena tolerancia. Es recomendable que su dispensación se haga sólo a través de receta médica, considerando que el mayor número de hombres que lo utilizan tienen otra patología de base y

\section{REFERENCIAS}

1. NIH Consensus Conference: Erectile Dysfunction. NIH Consensus Development Panel on ED. JAMA 1993; 270: 83-7.

2. Martin Morales A, Rosen R. Effective treatment of erectile dysfunction with Vardenafil. Drugs of Today 2003; 39: 51-9.

3. Gunter G, Trummer H. The etiology of erectile dysfunction and mechanisms by which drugs improve erection. Drugs of Today 2003; 39: 193201.

4. Coleman, Carabino J, Vergara C, Wang F. Vardenafil an oral selective PD inhibitor for the treatment of erectile dysfunction. Formulary 2003; 38: 131-48.

5. FERET B. Apomorphine a sublingual dopamine agonist for the treatment of erectile dysfunction. Formulary 2000; 35: 573-9.

6. Langtry $\mathrm{H}$, Markham A. Sildenafil, a review of its use in erectile dysfunción. Drugs 1999; 967-89. pudieran, por ende, estar utilizando otros medicamentos en forma simultánea, los cuales podrían interaccionar con Sildenafil aumentando su efecto vasodilatador ${ }^{7,10} \mathrm{y}$, por ende, sus efectos adversos.

7. AвRAmowicz M. Sildenafil: an oral drug for impotence. The medical letter 1998; 40: 51-2.

8. Goldstein, IRwin y cols. Vardenafil, a new phosphodiesterase type 5 inhibitor, in the treatment of erectile dysfunction in men with diabetes. Diabetes Care 2003; 26: 777-83.

9. Bradiey N, Christ J, Melman A. Sildenafil: a new oral therapy for erectile dysfunction. Drug of Today 1999; 35: 211-7.

10. HaRdMAn J, LimBiRd L. Goodman/Gilman Las Bases Farmacológicas de la Terapéutica, $10^{\mathrm{a}} \mathrm{Ed}$, Mc Graw Hill, México, 2003.

11. SweEtMan S. Martindale Guía Completa de Consulta Farmacoterapéutica, Pharma Editores, SL, Barcelona, 2003.

12. Cialis (tadalafil) in the management of erectile dysfunction. U.S Pharmacist 2004; 3-10.

13. Mc Crowe S, Streetman D. Vardenafil treatment for erectile dysfunction. The Annals of Pharmacotherapy 2004; 38: 77-85. 